\title{
Discovery of circular polarization in the Intermediate Polar 1WGA J1958.2+3232*
}

\author{
M. Uslenghi ${ }^{1}$, L. Tommasi ${ }^{2}$, A. Treves ${ }^{2}$, V. Piirola ${ }^{3}$, and P. Reig ${ }^{4,5}$ \\ 1 Istituto di Fisica Cosmica "G.Occhialini", CNR, Via Bassini 15, 20133 Milano, Italy \\ 2 Università degli Studi dell'Insubria, Polo di Como, Via Valleggio 11, 22100 Como, Italy \\ 3 Tuorla Observatory, SF21500 Piikkio, Finland \\ ${ }^{4}$ Foundation for Research and Technology - Hellas, 71010 Heraklion, Crete, Greece \\ ${ }^{5}$ Physics Department, University of Crete, 71003 Heraklion, Crete, Greece
}

Received 26 January 2001 / Accepted 31 March 2001

\begin{abstract}
We report on UBVRI polarimetry of the recently identified Intermediate Polar 1WGA J1958.2+3232, carried out on 2000 August, 4-6 at the Nordic Optical Telescope. Circular polarization was detected in $R$ and $I$ bands with an increasing absolute mean value with wavelength. There is evidence of possible modulation of the circular polarization at twice the previously reported white dwarf spin period, suggesting that it is the true period and that the modulation in optical and X-ray is dominated by the first harmonic. Indication of modulation at the orbital period is also present.
\end{abstract}

Key words. stars: individual: 1WGA J1958.2+3232 magnetic fields - polarization

\section{Introduction}

1WGA J1958.2+3232 is one of the objects found by Israel et al. (1998) in a systematic search for pulsators in the catalogue of ROSAT X-ray sources compiled by White et al. (1994). The source appeared modulated with a period $721 \pm 14 \mathrm{~s}$ and with a pulsed fraction of about $80 \%$. A subsequent observation performed by ASCA confirmed both the flux level and the strong periodic modulation at $734 \pm 1 \mathrm{~s}$ (Israel 2000, private communication), which was interpreted as a spin period.

On the basis of optical photometry and low resolution spectroscopy, Israel et al. (1999) proposed a $m_{V}=15.7$ star as the optical counterpart of 1WGA $\mathrm{J} 1958.2+3232$.

Further spectroscopic measurements carried out by Negueruela et al. (2000), as well as time resolved optical photometry performed by Uslenghi et al. (2000) (which provided detection of optical modulation at $733.24 \pm$ $0.02 \mathrm{~s}$ ), confirmed the candidate of Israel et al. (1999) and allowed its classification as an Intermediate Polar (IP).

Send offprint requests to: M. Uslenghi,

e-mail: uslenghi@ifctr.mi.cnr.it

* Based on observations made with the Nordic Optical Telescope, operated on the island of La Palma jointly by Denmark, Finland, Iceland, Norway and Sweden, in the Spanish Observatorio del Roque de los Muchachos of the Instituto de Astrofísica de Canarias.
A $4.36^{\mathrm{h}}$ modulation was also detected by Zharikov et al. (2000), in time-resolved photometric and spectroscopic observations, and interpreted as due to the orbital period.

We report here on the results of the first polarimetric measurements on this object.

\section{Observations}

The observations were carried out at the $2.56 \mathrm{~m}$ Nordic Optical Telescope (NOT), La Palma, equipped with the TURPOL double image chopping photopolarimeter (Piirola 1973, 1988). The instrument allows simultaneous measurements in the UBVRI bands and was operated in the simultaneous linear and circular polarization mode.

In this mode, polarization of the light is explored by a quarter-wave retarder plate, rotated through eight positions by $22.5^{\circ}$ steps. A calcite slab then splits the light into ordinary and extraordinary rays, that pass through identical diaphragms. Both components of sky background light enter in the two diaphragms, resulting in a cancellation of the polarization of sky light. Finally, the beam is split by four dichroic+bandpass filter combinations and sent to five photomultipliers. Each of these channels reproduces the spectral response of one of the UBVRI JohnsonCousins bands. In this way, truly simultaneous multiband observations can be performed. For all the observations, an integration time of $10 \mathrm{~s}$ was used for each position of 
Table 1. Journal of observations.

\begin{tabular}{cccc}
\hline \hline HJD & UT start & UT stop & n.measures \\
\hline $2451761^{\text {a }}$ & 01.03 .28 & 03.58 .47$. & 52 \\
2451762 & 22.26 .27 & 04.13 .12 & 76 \\
2451763 & 22.44 .40 & 04.34 .04 & 84 \\
\hline
\end{tabular}

a August 4, 2000.

Table 2. Circular polarization.

\begin{tabular}{cccc}
\hline \hline Band & 2000 Aug. 5 (\%) & 2000 Aug. 6 (\%) & Average (\%) \\
\hline$U$ & $-0.030 \pm 0.108$ & $+0.098 \pm 0.080$ & $+0.038 \pm 0.064$ \\
$B$ & $+0.078 \pm 0.144$ & $+0.209 \pm 0.104$ & $+0.164 \pm 0.084$ \\
$V$ & $+0.110 \pm 0.170$ & $+0.227 \pm 0.140$ & $+0.179 \pm 0.108$ \\
$R$ & $-0.535 \pm 0.128$ & $-0.553 \pm 0.106$ & $-0.546 \pm 0.082$ \\
$I$ & $-0.921 \pm 0.197$ & $-0.852 \pm 0.180$ & $-0.910 \pm 0.136$ \\
\hline
\end{tabular}

the retarder plate, giving a polarimetric measurement every $3.5 \mathrm{~min}$ (whereas the time resolution for photometry is about $23 \mathrm{~s}$ ). A $10 \mathrm{~s}$ sky background integration was normally performed every 15 min. High (HD 15445 and HD 204827) and null $\left(\mathrm{BD}+32^{\circ} 3739\right.$ and $\left.\mathrm{BD}+28^{\circ} 4211\right)$ polarization standard stars from Schmidt et al. (1992), observed many times per night (at the beginning, near midnight and at the end), were used to determine instrumental polarization and the orientation of the zero point of the retarder plate with respect to North.

We monitored 1WGA J1958.2+3232 for a total of about 13 hours, during three nights, on 2000 August 4, 5 and 6 (see the Journal of observations in Table 1). However, due to poor weather conditions, the signal-tonoise ratio during the first night is quite low and the data have been discarded.

\section{Data analysis and results}

Data reduction was performed using dedicated routines. They allow calculation of $Q, U$ and $V$ Stokes parameters by fitting the counts recorded in the eight positions of the retarder plate with a suitable cosine function, using a least-squares algorithm. Subtraction of a mean sky value obtained by interpolating between the nearest sky background acquisitions taken close to the object is automatically performed. Finally, the measured Stokes parameters are corrected by taking into account the instrumental constants, determined through observation of standard polarized stars. The error estimate takes into account both photon statistics and uncertainty of the least-squares fit.

Tables 2 and 3 report the two night means of circular and linear polarization, respectively. The former is characterized by amplitude and sign of the polarization percentage, the second by polarization percentage and position angle. Overall means of all the circular polarization collected data on both nights are reported in the last column of Table 2 .
Table 3. Linear polarization.

\begin{tabular}{ccccc}
\hline \hline & \multicolumn{2}{c}{ 2000 Aug. 5 } & \multicolumn{2}{c}{ 2000 Aug. 6 } \\
Band & $P(\%)$ & PA $\left(^{\circ}\right)$ & $P(\%)$ & PA $\left(^{\circ}\right)$ \\
\hline$U$ & $1.37 \pm 0.22$ & $141.2 \pm 4.5$ & $0.88 \pm 0.16$ & $143.2 \pm 5.2$ \\
$B$ & $1.53 \pm 0.29$ & $164.2 \pm 5.3$ & $0.84 \pm 0.21$ & $157.5 \pm 7.0$ \\
$V$ & $1.41 \pm 0.34$ & $148.1 \pm 6.8$ & $0.71 \pm 0.28$ & $114.7 \pm 10.7$ \\
$R$ & $1.06 \pm 0.26$ & $151.6 \pm 6.8$ & $0.97 \pm 0.21$ & $141.9 \pm 6.2$ \\
$I$ & $1.31 \pm 0.39$ & $6.9 \pm 8.4$ & $0.96 \pm 0.36$ & $104.6 \pm 10.3$ \\
\hline
\end{tabular}

\subsection{Wavelength dependence of circular polarization}

A strong wavelength dependence is apparent, with circular polarization increasing, in absolute value, toward the infrared. This is a common result for polarized IPs (BG CMi, Penning et al. 1986; PQ Gem, Piirola et al. 1993; RX J1712.6-2414, Buckley et al. 1997) and it is generally attributed to cyclotron emission, possibly in combination with free-free emission (Chanmugam \& Frank 1987; West et al. 1987; Piirola et al. 1993; Väth 1997; Buckley 2000). Cyclotron radiation produces very large circular polarization and the emission is strong at low harmonics, but drops suddenly at higher harmonics. The drop is used to estimate the strength of the magnetic field: typical values estimated for the polarized Intermediate Polars are in the range 5-10 MG (see, e.g., Piirola et al. 1993), below the 10-100 MG reported for the Polars.

In Fig. 1 the observed polarized fluxes $\left(F_{\mathrm{c}}=P_{\mathrm{c}} F_{\text {tot }}\right)$ are reported for the $R$ and $I$ bands, together with the $3 \sigma$ upper limits in $U B V$, versus $\omega / \omega_{\mathrm{c}}$, where $\omega_{\mathrm{c}}$ is the cyclotron frequency for a typical magnetic field of $8 \mathrm{MG}$. Normalization is arbitrary for both axis but, since the scale is logarithmic, changes in normalization result in a shift only.

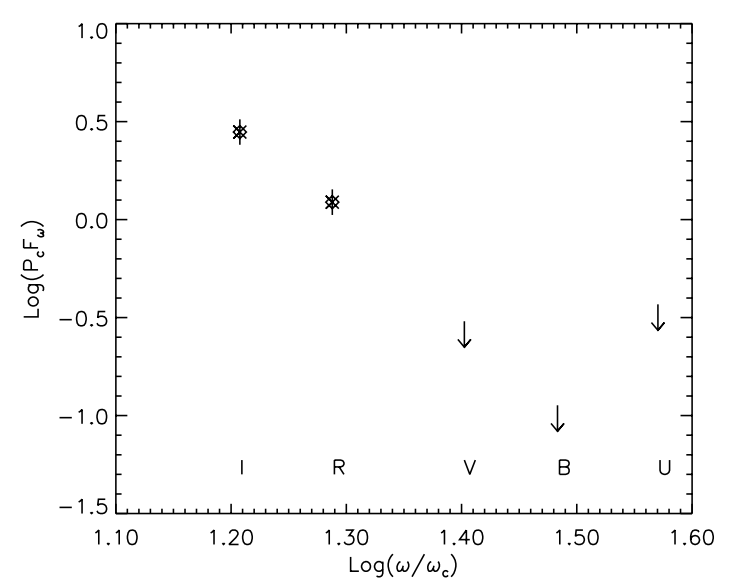

Fig. 1. Wavelength dependence of the circularly polarized fluxes; $\omega_{\mathrm{c}}$ is referred to a $8 \mathrm{MG}$ field. Each point is calculated from the overall observations. 


\subsection{Polarimetric variability}

Polarimetric data were analysed for the presence of periodic variations related to the dynamics of the binary system.

\subsubsection{Circular polarization}

In Fig. 2 the Lomb-Scargle periodogram (Scargle 1982) of the degree of the circularly polarized light in $U B V R I$ bands is reported. $\Omega$ and $\omega$ are the purported orbital and white dwarf spin frequencies, respectively $\left(\Omega^{-1}=4.36 \mathrm{~h}\right.$, Zharikov et al. $2000, \omega^{-1}=733.2 \mathrm{~s}$, Uslenghi et al. 2000). No evidence of a periodicity at $\omega$ is present. However, some power around half of this frequency can be seen in all bands other than $U$. The clearest evidence is in the $I$ filter, where the second highest peak in the periodogram occurs at $1464 \pm 7 \mathrm{~s}$, consistent with $\omega / 2$. Since the frequency is known a priori, in computing the significance level for this peak the correction for multiple trials does not apply and the significance level, due to the exponential distribution of the power at a given frequency, is simply given by $1-\mathrm{e}^{-z}$, where $z$ is the value of the periodogram (i.e. the power normalized by the variance). In this case $(z=6.6)$ the significance level is $99.8 \%$ (in effect, the presence of non-white noise results in a significance level lower than the theoretical one). Moreover, the highest peak is found at an aliased frequency of $\omega / 2$, produced by the sampling pattern of the polarimeter, as apparent in the spectral window (bottom panel in Fig. 2). This raises the possibility that the true period is $1466 \mathrm{~s}$, while the previously reported spin periods (e.g. from the $\mathrm{X}$-rays) are the first harmonic.

Figure 3 shows the $I$-band data folded on the $1466 \mathrm{~s}$ period and rebinned. Modulation is apparent, with peakto-peak amplitude of about $2 \%$ and mean value of about $-1 \%$.

Modulation at the orbital period and at its first harmonic is also present in the $R$ band. The data folded over the orbital period are presented in Fig. 4.

\subsubsection{Linear polarization}

Due to the reduced efficiency $(50 \%)$ of the linear polarization measurements in the simultaneous linear and circular polarization mode, the data have a lower signal to noise ratio than for the circular polarization. A Lomb-Scargle periodograms of the $Q / I$ and $U / I$ normalized Stokes parameters does not show any significant peak. Near the frequencies of interest, we carried out a $\chi^{2}$ epoch folding analysis (Leahy et al. 1983), which is more sensitive than the Fourier techniques to non-sinusoidal signals, but no significant features were apparent.

The relatively flat wavelength dependence of the average linear polarization (Table 3 ) is within the measurement errors consistent with that of typical interstellar polarization, and there is no clear evidence of intrinsic component from the nightly mean polarization values.

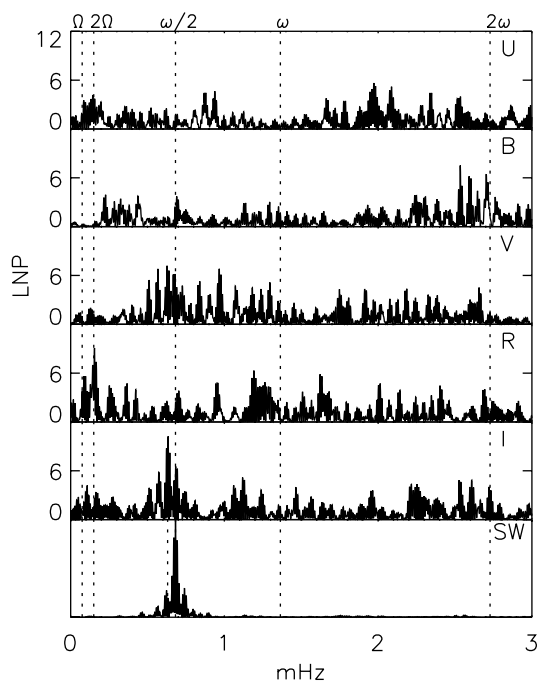

Fig. 2. Lomb-Scargle periodogram of the circular polarization data in all bands. In the bottom panel, the spectral window is shown (it has been shifted in order to have the peak at $\omega / 2$ ).

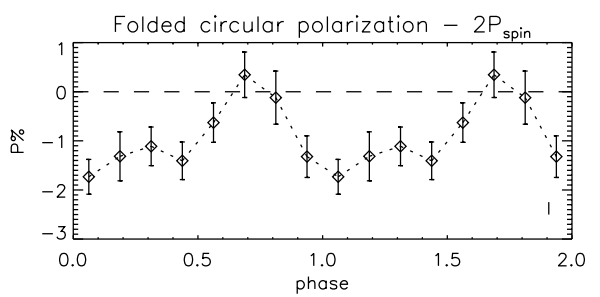

Fig. 3. Circular polarization data in $I$ band, folded with the $1466 \mathrm{~s}$ period and rebinned. The time of the zero phase for all the folded plot in this paper is HJD $=2451761^{\mathrm{d}} 2281$ (Zharikov et al. 2000).

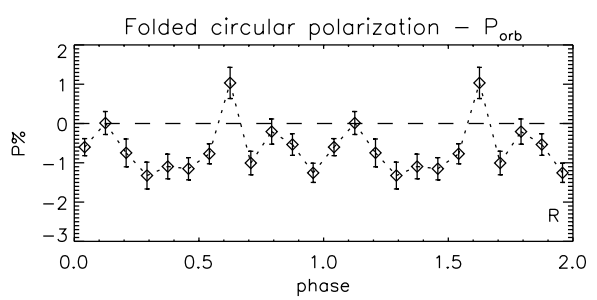

Fig. 4. Circular polarization data in $R$ band, folded with the orbital period $\left(4.36^{\mathrm{h}}\right.$, Zharikov et al. 2000$)$, and rebinned. The time of the zero phase is HJD $=2451761$. 2281 .

\subsection{Photometry}

Photometric data were first corrected for atmospheric extinction and then a 3rd order polynomial was subtracted in order to remove long time scale variations due to residual atmospheric effects. Periodograms of the flux intensity in the five bands are presented in Fig. 5. In $U$ band a well defined peak occurs at $\omega^{-1}=733.2 \pm 0.1 \mathrm{~s}$ and synodic pulses at both $\omega-\Omega$ and $\omega+\Omega$ are clearly apparent. In the other bands the $\omega$ peak is weaker, but some power concentration is always present. Modulation at the orbital period $\left(\Omega^{-1}=4.46 \pm 0.1 \mathrm{~h}\right.$, with $1 / \mathrm{d}$ alias at 3.76 and $\left.5.48 \mathrm{~h}\right)$ and at its first and second harmonic is also detected. Instead, 
we cannot find any indication of the $1466 \mathrm{~s}$ period and light curves folded at this period show no significant oddeven effect. If the spin period is indeed $1466 \mathrm{~s}$, both poles must emit in a very symmetrical manner.

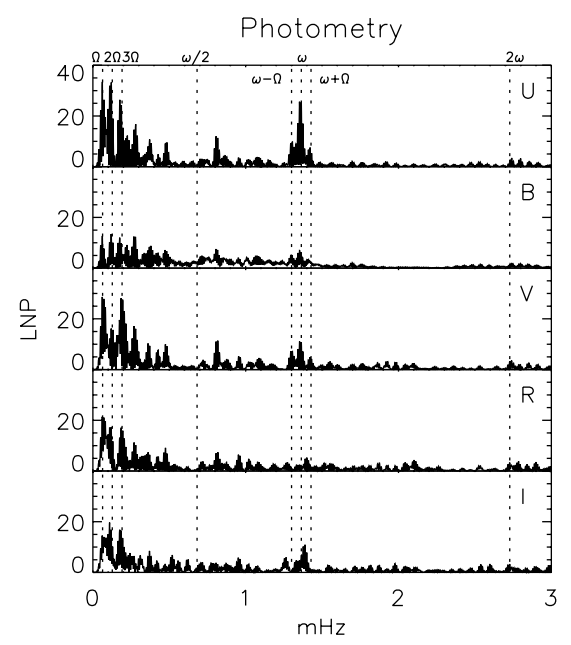

Fig. 5. Lomb-Scargle periodogram of the flux in $U B V R I$ bands.

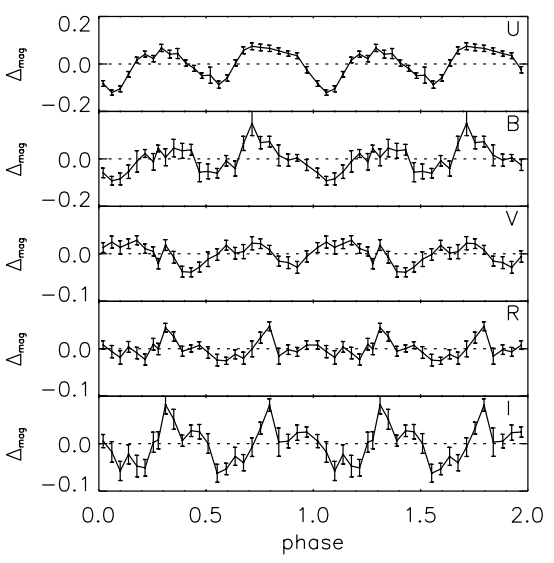

Fig. 6. Folded light curves at the 1466 s period with 25 bin, in magnitudes relative to the mean level. The standard errors of the bin values were calculated from the scatter of the individual integrations (in this case 40).

\section{Discussion}

Polarization curves and measurements of cyclotron lines are used to measure the magnetic field and the topology in Polars. Instead, in Intermediate Polars, which have lower magnetic fields, polarization is rarely detected. To our knowledge, only four other IPs show significant polarization: BG CMi (Penning et al. 1986), PQ Gem (Piirola et al. 1993), RX J1712.6-2414 (Buckley et al. 1997) and V405 Aur (Shakhovskoj \& Kolesnikov 1997;
Piirola et al. 2001). The circular polarization of BG Cmi appears to be constant, whereas the latter three show spin modulation: in all cases the polarization is in the order of few $\%$.

Our data are not sufficient to establish conclusively phase-dependent variability of the polarization at any of the characteristic frequencies, but, surprisingly, the clearest indication is for a modulation at a period twice the accepted spin period. This seems provide a strong evidence that the spin period is $1466 \mathrm{~s}$ and that the main frequency in optical and X-ray light curves is its first harmonic. In Uslenghi et al. (2000) evidence for optical flux modulation at the $1466 \mathrm{~s}$ period was reported, but for one night only. However, among the IP, there are other systems (YY Dra and V405 Aur, Allan et al. 1996) which have optical and $\mathrm{X}$-ray light curves dominated by the first harmonic.

Acknowledgements. We thank the NOT staff for technical support during the observations, in particular Anlaug Amanda Kaas and Carlos Perez. We are grateful to Lucio Chiappetti for helpful conversations and to Santo Catalano for a critical reading of the manuscript and for his valuable comments. Financial support from EC grant ERBFM-RXCT 98-0195 and Italian Murst COFIN 98021541 are acknowledged.

\section{References}

Allan, A., Horne, K., Hellier, C., et al. 1996, MNRAS, 279, 1345

Buckley, D. A. H., Haberl, F., Motch, C., et al. 1997, MNRAS, 287, 117

Buckley, D. A. H. 2000, NewAR, 44, 63

Chanmugam, G., \& Frank, J. 1987, ApJ, 320, 746

Israel, G. L., Angelini, L., Campana, S., et al. 1998, MNRAS, 298, 502

Israel, G. L., Covino, S., Polcaro, V. F., \& Stella, L. 1999, A\&A, 345, L1

Leahy, D. A., Darbro, W., Elsner, R. F., \& Weisskopf, M. C. 1983, ApJ, 266, 160

Negueruela, I., Reig, P., \& Clark, J. S. 2000, A\&A, 354, L29

Penning, W. R., Schmidt, G. D., \& Liebert, J. 1986, ApJ, 301, 881

Piirola, V. 1973, A\&A, 27, 383

Piirola, V. 1988, in Polarized radiation of circumstellar origin, ed. G. V. Coyne et al. (University of Arizona Press), 735

Piirola, V., Hakala, P., \& Coyne, G. V. 1993, ApJ, 410, L107

Piirola, V., et al. 2001, in preparation

Scargle, J. D. 1982, ApJ, 263, 835

Schmidt, G. D., Elston, R., \& Lupie, O. L. 1992, AJ, 104, 1563

Shakhovskoj, N. M., \& Kolesnikov, S. V. 1997, IAUC 6760

Uslenghi, M., Bergamini, P., Catalano, S., Tommasi, L., \& Treves, A. 2000, A\&A, 359, 639

Väth, H. 1997, A\&A, 317, 476

West, S. C., Berriman, G., \& Schmidt, G. D. 1987, ApJ, 322, L35

White, N. E., Giommi, P., \& Angelini, L. 1994, IAU Circ. 6100

Zharikov, S. V., Tovmassian, G. H., Echevarría, J., \& Cárdenas, A. A. 2001, A\&A, 366, 834 